\title{
Metabolomics Society 2014 Metabolomics Publication Awards
}

\author{
Roy Goodacre
}

Published online: 21 August 2014

(C) Springer Science+Business Media New York 2014

In 2013, the Board of the Directors of the Metabolomics Society commissioned a new award scheme to recognise the excellent research published in the journal Metabolomics. Each year, the Society will recognise the top papers within the following categories:

- Best Paper Award-for the paper with the highest total number of citations during the previous three years. The first author of the winning paper receives a gift certificate from Springer, a certificate from the President of the Society, a front cover image in the Metabolomics journal, and the paper will be made open access for 30 days. The first author of the runnerup paper receives a certificate from the President of the Society.

- Highest Download Award-for the paper with the highest total number of downloads during the previous three years. The first authors of the winning and runnerup papers each receive a certificate from the President of the Society.

Only primary research papers and "best practice" papers are eligible for these publication awards, which will be announced annually at the international Metabolomics Society conference, typically held in June. Congratulations to the following 2014 winners!

\section{Best Paper Award}

- Winner-Koek, M.M., van der Kloet, F.M., Kleemann, R., Kooistra, T., Verheij, E.R. \& Hankemeier, T. (2011)
Semi-automated non-target processing in $\mathrm{GC} \times \mathrm{GC}-\mathrm{MS}$ metabolomics analysis: applicability for biomedical studies. Metabolomics 7(1), 1-14, http://link.springer. com/article/10.1007\%2Fs11306-010-0219-6 DOI 10. 1007/s11306-010-0219-6.

- Runner up-Ivanišević, J., Thomas, O.P., Lejeusne, C., Chevaldonné, P. \& Pérez, T. (2011) Metabolic fingerprinting as an indicator of biodiversity: towards understanding inter-specific relationships among Homoscleromorpha sponges. Metabolomics 7(2), 289-304, http://link.springer.com/article/10.1007\%2Fs11306010-0239-2 DOI 10.1007/s11306-010-0239-2.

\section{Highest Download Award}

- Winner-Wahyuni, Y., Ballester, A.-R., Tikunov, Y., de Vos, R.C.H., Pelgrom, K.T.B., Maharijaya, A., Sudarmonowati, E., Bino, R.J. \& Bovy, A.G. (2013) Metabolomics and molecular marker analysis to explore pepper (Capsicum sp.) biodiversity. Metabolomics 9(1), 130-144, http://link.springer.com/arti cle/10.1007/s11306-012-0432-6 DOI 10.1007/S11306012-0432-6.

- Runner up-Theodoridis, G., Gika, H., Franceschi, P., Caputi, L., Arapitsas, P., Scholz, M., Masuero, D., Wehrens, R., Vrhovsek, U. \& Mattivi, F. (2012) LC-MS based global metabolite profiling of grapes: solvent extraction protocol optimisation. Metabolomics 8(2), 175-185, http://link.springer.com/article/10.1007/ s11306-011-0298-z DOI 10.1007/S11306-011-0298-Z.

R. Goodacre $(\square)$

School of Chemistry, University of Manchester, Manchester, UK

e-mail: roy.goodacre@manchester.ac.uk 